\title{
Enasidenib: a magic bullet for myelodysplastic syndromes?
}

In The Lancet Haematology, Eytan M Stein and colleagues $^{1}$ present a subgroup analysis of a published multicentre phase 1-2 trial (AG221-C-001) of the oral drug enasidenib in relapsed or refractory acute myeloid leukaemia and myelodysplastic syndromes that harbour mutations in the isocitrate dehydrogenase-2 (IDH2) gene. $^{2}$ In their subgroup analysis, the outcomes of the few myelodysplastic syndrome cases included in AG221-C-001 were extracted from the bulk of the data and described in detail. 17 patients with advanced myelodysplastic syndromes who were in relapse or refractory to previous treatments received enasidenib, a specific inhibitor of mutant IDH2, as salvage therapy. The paucity of cases examined was a result of the low incidence of mutations affecting $\mathrm{IDH} 2$ in myelodysplastic syndromes (detected in around $5 \%$ of cases). ${ }^{3}$ Nevertheless, the presentation of the results in myelodysplastic syndromes separated from those in acute myeloid leukaemia, despite the limited number of observations, is important and might indeed modify the standard of care.

Enasidenib was approved in 2017 by the US Food and Drug Administration for the treatment of relapsed or refractory, IDH2-mutated acute myeloid leukaemia. Myelodysplastic syndromes are often considered to be in continuum with acute myeloid leukaemias, but in fact are molecularly and pathogenetically different. ${ }^{4}$ Epigenetic dysregulation and abnormalities in these two diseases are not identical ${ }^{4}$ and, although the two conditions share several somatic gene mutations, the outcomes of the same therapies are not always equal, as a result of the different biology and clinical presentations. This variance in outcomes is why the results of Stein and co-workers deserve particular attention while we await the results of ongoing clinical studies of enasidenib (NCT03744390, NCT03383575, and NCT03839771) in myelodysplastic syndromes, including final results of the AG221-C-001 trial (NCT01915498).

The rare IDH2 mutations affect methylation processes and epigenetic regulation, determine production of the oncometabolite R-2-hydroxyglutarate, and confer poor prognosis in myelodysplastic syndromes. ${ }^{5}$ Few treatment options are available for patients with myelodysplastic syndromes in whom therapy (mainly with hypomethylating drugs) has failed, and survival after treatment failure is extremely short, both for low-risk and high-risk cases. ${ }^{6}$ The treating physician is left with difficult decisions in the absence of effective drugs. Therefore, the number of overall responses observed by Stein and colleagues, in more than a half of patients $(n=9[53 \%])$ is extremely relevant. The patients described received several lines of therapy, including haematopoietic stem cell transplant, and represented a group with particularly dismal prognosis. ${ }^{6}$ Although the small number of cases prompts caution in the final judgment of these preliminary results, the improvement in overall survival, from around 5 months usually reported in the literature for relapsed or refractory myelodysplastic syndrome cases, ${ }^{6}$ to a median of 16.9 months $(95 \% \mathrm{Cl} 1.5-32.3)$ in their study, is impressive.

The applicability of target therapy in myelodysplastic syndromes depends on the extent of molecular characterisation, not only at the onset of disease to determine initial prognosis, but also during follow-up, and on failure of any line of treatment. The identification of acquired mutations or their clonal expansion might allow new targeted treatment approaches, such as with enasidenib.? Given the increasing availability and expansion of molecular testing, haematologists should keep molecular characterisation in mind when re-evaluating patients to select a rescue therapy.

The authors observed that enasidenib-responsive patients carried fewer co-mutations in addition to mutation in $I D H 2$. The prognostic weight of the number of concurrent mutations on overall survival is established in myelodysplastic syndromes; ${ }^{8}$ however, the effect of co-mutations on response to various treatments, although suggested for some drugs, is controversial and needs to be further clarified.

The clonal architecture of myelodysplastic syndromes is modified during the natural history of the disease and determines its progression. How these modifications influence or correlate with response to therapy is a subject that needs further investigation. An improved understanding of the relative importance of single mutations, their interactions when co-expressed, and the importance of allele burden is the aim of future studies. In the present work, changes in the variant

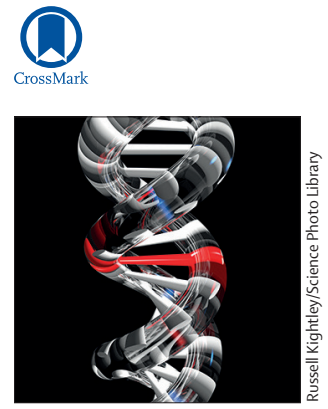

Lancet Haematol 2020 Published Online March 5, 2020 https://doi.org/10.1016/ S2352-3026(20)30039-9

See Online/Articles https://doi.org/10.1016/ S2352-3026(19)30284-4 
allele frequency of mutated IDH2 during therapy were not reported. If and how variant allele frequency is modulated could be a clue to clarifying the mechanism of action and efficacy of enasidenib.

In their cohort of patients with advanced myelodysplastic syndromes, Stein and colleagues found that enasidenib was not able to eradicate disease, which could have been related to the expansion of other clones present, limiting the efficacy of monotherapy with enasidenib, ${ }^{9}$ although other mechanisms of resistance have also been described. ${ }^{10}$

For decades, researchers have aimed at finding socalled magic bullet therapies against haematological malignancies. They are now in the position to evaluate the positive results of specific, molecularly targeted drugs, beginning with all-trans retinoic acid, and subsequently progressing to imatinib, ibrutinib, and midostaurin, among others. Particularly in acute myeloid leukaemia and myelodysplastic syndromes, the idea of successfully removing the maturation blockage and inducing cell differentiation has been a long-standing theme in preclinical and clinical studies. With enasidenib, this differentiation has been achieved in acute myeloid leukaemia. ${ }^{2}$ In their study, Stein and colleagues showed that rapid differentiation of fast and long-lasting effect is also obtainable in IDH2-mutated myelodysplastic syndromes, which was reflected in the improvement in survival.

Enasidenib combines adequate tolerability, oral administration, and high activity into a single therapy. Stein and colleagues showed, for the first time, that this monotherapy is able to achieve a response, at least for a notable period, in the rare cases of myelodysplastic syndromes with mutations in IDH2. ${ }^{1}$ Possible combination with other active drugs will provide new and promising perspectives in the treatment of this small, but prognostically disadvantaged, subgroup of patients with myelodysplastic syndromes.

I have received honoraria for lecturing and participation on advisory boards in the past three years from Agios, Amgen, Astex, Celgene, Janssen, Menarini, Novartis, and Takeda.

\section{Valeria Santini}

santini@unifi.it

Myelodysplastic Syndrome Unit, Hematology Division, Azienda OspedalieroUniversitaria Careggi, University of Florence, 50134 Florence, Italy

1 Stein EM, Fathi AT, DiNardo CD, et al. Enasidenib in patients with mutant IDH2 myelodysplastic syndromes: a phase 1 subgroup analysis of the multicentre AG221-C-001 trial. Lancet Haematol 2020; published online March 5. https://doi.org/10.1016/S2352-3026(19)30284-4

2 Stein EM, DiNardo CD, Pollyea DA, et al. Enasidenib in mutant IDH2 relapsed or refractory acute myeloid leukemia. Blood 2017; 130: 722-31.

3 Pardanani A, Patnaik MM, Lasho TL, et al. Recurrent IDH mutations in high-risk myelodysplastic syndrome or acute myeloid leukemia with isolated del(5q). Leukemia 2010; 24: 1370-72.

4 Figueroa ME, Skrabanek L, LiY, et al. MDS and secondary AML display unique patterns and abundance of aberrant DNA methylation. Blood 2009; 114: 3448-58.

5 DiNardo CD, Jabbour E, Ravandi F, et al. IDH1 and IDH2 mutations in myelodysplastic syndromes and role in disease progression. Leukemia 2016; 30: 980-84

6 Prébet T, Gore SD, Esterni B, et al. Outcome of high-risk myelodysplastic syndrome after azacitidine treatment failure. J Clin Oncol 2011; 29: 3322-27.

7 Santini V. How I treat MDS after hypomethylating agent failure. Blood 2019; 133: 521-29.

8 Papaemmanuil E, Gerstung M, Malcovati L, et al. Clinical and biological implications of driver mutations in myelodysplastic syndromes. Blood 2013; 122: 3616-27

9 Quek L, David MD, Kennedy A, et al. Clonal heterogeneity of acute myeloid leukemia treated with the IDH2 inhibitor enasidenib. Nat Med 2018 24: 1167-77.

10 Intlekofer AM, Shih AH, Wang B, et al. Acquired resistance to IDH inhibition through trans or cis dimer-interface mutations. Nature 2018; 559: 125-29. 